\title{
Technical and safety performance of CT-guided percutaneous microwave ablation for lung tumors: an ablate and resect study
}

\author{
Shanda H. Blackmon ${ }^{1}$, Rosalie M. Sterner ${ }^{1 \wedge}$, Patrick W. Eiken², Thomas J. Vogl ${ }^{3}$, Bradley B. Pua ${ }^{4}$, \\ Jeffrey L. Port ${ }^{5}$, Damian E. Dupuy ${ }^{6}$, Matthew R. Callstrom ${ }^{2}$
}

${ }^{1}$ Division of General Thoracic Surgery, Mayo Clinic, Rochester, MN, USA; ${ }^{2}$ Department of Radiology, Mayo Clinic, Rochester, MN, USA; ${ }^{3}$ Department of Diagnostic and Interventional Radiology, University Hospital Frankfurt am Main, Frankfurt am Main, Germany; ${ }^{4}$ Division of Interventional Radiology, Department of Radiology, Weill Cornell Medicine, New York, NY, USA; ${ }^{5}$ Department of Cardiothoracic Surgery, Weill Cornell Medicine, New York-Presbyterian Hospital, New York, NY, USA; ${ }^{6}$ Department of Diagnostic Imaging, the Warren Alpert Medical School of Brown University, Providence, RI, USA

Contributions: (I) Conception and design: SH Blackmon, PW Eiken; (II) Administrative support: None; (III) Provision of study materials or patients: SH Blackmon, PW Eiken, TJ Vogl, BB Pua, JL Port, DE Dupuy, MR Callstrom; (IV) Collection and assembly of data: SH Blackmon, PW Eiken, TJ Vogl, BB Pua, JL Port, DE Dupuy, MR Callstrom; (V) Data analysis and interpretation: SH Blackmon, RM Sterner, PW Eiken; (VI) Manuscript writing: All authors; (VII) Final approval of manuscript: All authors.

Correspondence to: Shanda H. Blackmon, MD, MPH. Professor of Surgery, Division of General Thoracic Surgery, Mayo Clinic, 200 1st St., SW, Rochester, MN 55905, USA. Email: Blackmon.shanda@mayo.edu.

Background: Percutaneous image-guided thermal ablation has an increasing role in the treatment of primary and metastatic lung tumors. Achieving acceptable clinical outcomes requires better tools for preprocedure prediction of ablation zone size and shape.

Methods: This was a prospective, non-randomized, single-arm, multicenter study conducted by Medtronic (ClinicalTrials.gov ID: NCT02323854). Subjects scheduled for resection of metastatic or primary lung nodules underwent preoperative percutaneous microwave ablation. Ablation zones as measured via CT imaging following ablation immediately and before resection surgically versus predicted ablation zones as prescribed by the investigational system software were compared. This CT scan occurred after the ablation was finished but the antenna still in position. Time (minutes) from antenna placement to removal was 23.7 \pm 13.1 ( $n=14$ ); median: 21.0 (range, 6.0 to 48.0). The definition of the secondary endpoint of complete ablation was $100 \%$ non-viable tumor cells based on nicotinamide adenine dinucleotide hydrogen (NADH) staining. Safety endpoints were type, incidence, and severity of adverse events.

Results: Fifteen patients (mean age 58.9 years; $67 \%$ male; 33\% female) were enrolled in the study, $33.3 \%$ (5/15) with previous thoracic surgery, 73\% (11/15) with metastasis, and 27\% (4/15) with primary lung tumors. All underwent percutaneous microwave ablation followed by surgical resection the same day. Complete ablation was detected in 54.4\% (6/11), incomplete ablation in 36.4\% (4/11), and delayed necrosis in $9.1 \%$ (1/11). There were no device-related adverse events. Ablation zone volume was overestimated in all patients.

Conclusions: Histological complete ablation was observed in 55\% of subjects. CT scanning less than an hour after ablation and tissue shrinkage may account for the smaller zone of ablation observed compared to predicted by the investigational system software.

Keywords: Lung cancer; ablation; lung ablation; metastectomy; metastasis; microwave ablation

Submitted Apr 05, 2021. Accepted for publication Oct 22, 2021.

doi: $10.21037 /$ jtd-21-594

View this article at: https://dx.doi.org/10.21037/jtd-21-594

^ ORCID: 0000-0002-9323-3625. 


\section{Introduction}

Surgery is the gold standard for the treatment of lung tumors, but many patients are inoperable due to comorbidities or diminished pulmonary reserve. Thus, alternative treatments, such as percutaneous image-guided thermal ablation have been used $(1,2)$. The main types of ablation technologies used in the lung are radiofrequency ablation, cryoablation, and microwave ablation (2). Potential strengths of microwave energy in the lung include more reliable heat deposition in aerated lung parenchyma compared to radiofrequency ablation (3-14). In addition, microwave ablation offers less susceptibility to local cooling from pulmonary blood vessels and shorter procedure times than cryoablation or radiofrequency ablation (4,9,15-19). The main weakness of microwave ablation in the lung is the unpredictable size of the ablation zone, which can create challenges for the operator in confidently achieving adequate margins while avoiding critical structures or unnecessarily large ablation zones. In order to maximize ablation efficacy while minimizing collateral damage to normal tissue, it is necessary to develop better tools to predict ablation zone size as a function of time and energy delivered.

In this study, the Emprint ${ }^{\mathrm{TM}}$ procedure planning application was used to predict ablation zone size. The Emprint ${ }^{\mathrm{TM}}$ procedure planning application is an interactive tool that predicts the size of a microwave ablation zone based on time, energy, and target tissue type and represents that data visually on pre- or intra-procedure CT images. This feedback is intended to allow the operator to adjust probe position, energy delivered, or duration of ablation to maximize the efficacy of the ablation procedure, but clinical validation of the software's accuracy is required. The primary objective of this study was to evaluate the technical performance of the Emprint ${ }^{\mathrm{TM}}$ system in predicting the size of ablation zones. Inclusion/exclusion criteria and study recruitment are depicted in Figure 1. The Emprint ${ }^{\mathrm{TM}}$ ablation system is indicated for use in the United States in percutaneous, laparoscopic, and interoperative coagulation (ablation) of soft tissue, including partial or complete ablation of non-resectable liver tumors (not for use in cardiac procedures). The primary endpoint was dose response-how well the size of the ablation zone predicted by the software matched the actual size of the ablation zone created. Dose response was assessed by comparing the predicted size of ablation zones as determined by the Emprint $^{\mathrm{TM}}$ procedure planning application versus the actual ablation zone size as observed on immediate post-ablation CT. Histology was used for the secondary endpoint, which was complete ablation for each target tumor defined as $100 \%$ non-viable tumor cells on NADH viability staining. Safety endpoints included type, incidence, and severity of adverse events.

We present the following article in accordance with the TREND reporting checklist (available at https://dx.doi. org/10.21037/jtd-21-594).

\section{Methods}

This was a prospective, non-randomized, single-arm, multicenter study designed to evaluate the Emprint ${ }^{\mathrm{TM}}$ ablation system via a percutaneous approach in patients with metastatic or primary lung tumors (ClinicalTrials.gov ID: NCT02323854). The study was conducted in accordance with the Declaration of Helsinki (as revised in 2013). The study was approved by institutional review board of the participating institutions (IRB approval: 15-001772) on July 9, 2015 and informed consent was taken from all the patients.

All subjects meeting the inclusion/exclusion criteria were enrolled into the study. The study ran from January 2015-March 2017. Ablations were carried out using a percutaneous approach at a dose (power and time setting) selected during procedure planning. A commercially available Medtronic software application (Emprint Procedure Planning Application, CAVA, Medtronic, Boulder, CO, USA) was used as an adjunct to assist with planning for the ablation procedure in subjects already scheduled for surgical resection of metastatic or primary lung disease per standard practice (Figure 2). During the product design and development phases, the Emprint ${ }^{\mathrm{TM}}$ Ablation System was tested using both ex vivo and in vivo testing models $(3-18,20)$. These data were used to define the ablation zone size characteristics based on energy and time dose, available in the antenna instructions for use (3-18,20). An in vivo study was conducted using a porcine model to evaluate the performance of the Emprint ${ }^{\mathrm{TM}}$ Ablation System in terms of ablation zone size (16). Variable power and time settings were evaluated to capture a range of ablation zone sizes in the lung. Outcomes of the study included gross measurements of the ablation zones postmortem as well as ablation zone size measurements from CT imaging. The Emprint ${ }^{\mathrm{TM}}$ Ablation System was evaluated at 45,75 , and $100 \mathrm{~W}$ and a range of time settings (2:20-10:00 minutes) in lung tissue of domestic swine (16). 


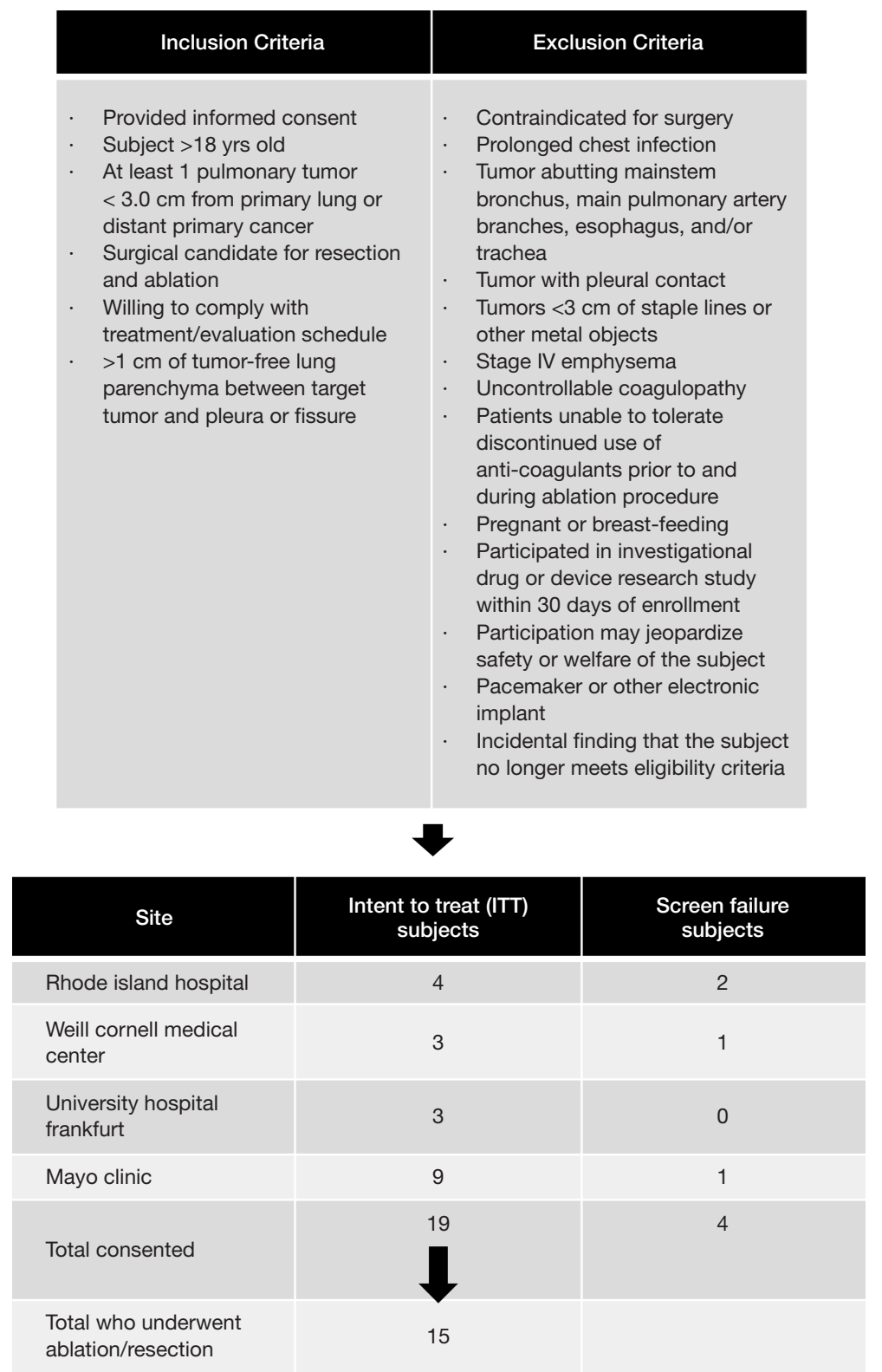

Figure 1 Inclusion/exclusion criteria and study recruitment. Inclusion and exclusion criteria are depicted followed by an outline of recruitment process by site.

After surgical access and ablation zone creation, a subset of target zones underwent unenhanced CT scans performed with or without the antenna in place (16). The Emprint ${ }^{\mathrm{TM}}$ Procedure Planning Application is guidance software that utilizes pre-procedural computed tomography (CT) images to visualize predicted ablation zones (3-18,20). The predicted ablation zone displayed in the software is derived from the lung-specific in vivo data collected in the studies described above (16). The physician prescribed the dose then overlaid a virtual predicted ablation zone relative to the target tumor on the patient CT images using the application (Figure 2). This included planning the dose to the active zone and the passive zone. In each patient, a single tumor was ablated using a single placed probe. Once the ablation procedure was completed, the patient remained intubated on single lung ventilation and scheduled surgical 

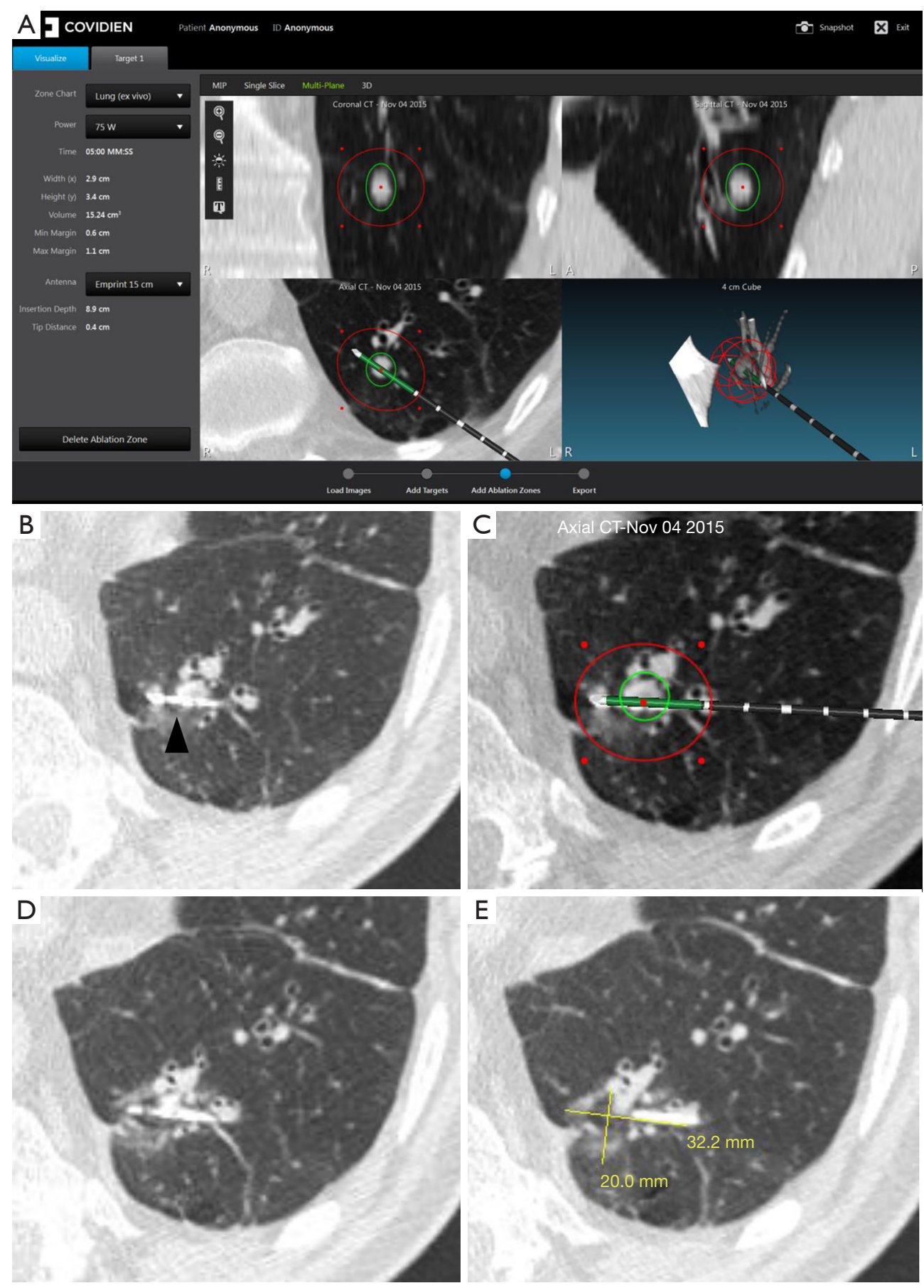

Figure 2 Microwave ablation of an $8 \mathrm{~mm}$ colon cancer metastasis in the left lower lobe. Immediately before probe placement, CT images were obtained and loaded into the Emprint ${ }^{\mathrm{TM}}$ ablation planning system. (A) Software displays planned probe placement (black line), target lesion (green ovals), and anticipated ablation zone (red ovals) in a multiplanar format. (B) Axial CT image obtained after placement of $15 \mathrm{~cm} \mathrm{Emprint}^{\mathrm{TM}}$ probe (arrowhead). (C) Axial screen capture from Emprint ${ }^{\mathrm{TM}}$ software showing revised plan with predicted ablation zone (red oval) based on actual needle placement shown in B. (D) Axial CT image obtained after 5-minute ablation at 75 watts with probe still in place. (E) Axial CT image demonstrating measurement of ground glass opacity representing the ablation zone. 
tumor resection was performed. Dose response was assessed by the comparison between actual versus predicted ablation zone size with volume (measured for each ablation zone via CT imaging immediately following ablation and before surgical resection) was used to assess dose response. This CT scan was obtained once the ablation was finished but with the antenna still in position. Time (in minutes) from antenna placement to removal was $23.7 \pm 13.1(\mathrm{n}=14)$; median: 21.0 (range, 6.0 to 48.0). CT assessment of the ablation was based on ground glass opacity. A minimum of three board-certified radiologists were trained and assigned to review images for this study. During the processing of the images, all images were blinded so that radiologists did not have access to any patient confidential information and local site assessments. The cases were loaded onto the software for review by two independent radiologists. Response was measured for each ablation via CT imaging acquired right after the ablation but with the antenna still in place. CT measurements of the ablation zone immediately post-procedure with the antenna in place was compared to the predicted ablation zones prescribed by the physician during planning. Sites also acquired a final CT image after the antenna has been removed to assess for any adverse events that may have resulted from the ablation procedure. Post-procedure imaging acquired after antenna removal was not reviewed by an independent third party to the sponsor. Following the procedure, histology was used to assess tumor ablation. Complete ablation was defined as $100 \%$ non-viable tumor cells based on nicotinamide adenine dinucleotide hydrogen (NADH) staining. Safety endpoints included type, incidence, and severity of adverse events. Adverse event data were collected starting from the initial administration of anesthesia until conclusion of the first post-operative follow-up visit. An image of a gross specimen, ablation zone, and tumor are shown in Figure S1 and an outline of the methodology is shown in Figure S2.

Patients were recruited at Mayo Clinic, University Hospital Frankfurt am Main, Weill Cornell Medicine, and the Warren Alpert Medical School of Brown University. A total of 19 subjects consented to participate in the study, and ablation was completed for 15 subjects, of which 15 (100\%) completed the study. There were primary and secondary endpoint results available for 11 subjects. $33.3 \%(5 / 15)$ of subjects had a history of thoracic surgery, $73 \%(11 / 15)$ had metastatic tumors, and 27\% (4/15) had primary tumors.

Inclusion criteria were: informed consent given by subject or authorized representative, subject $\geq 18$ years of age, there was at least one pulmonary metastasis $\leq 3.0 \mathrm{~cm}$ in maximum diameter from a distant primary cancer or one primary lung cancer $\leq 3.0 \mathrm{~cm}$ in maximum diameter, a thoracic surgeon determined the subject is a candidate for resection of the tumor targeted for ablation, subject is willing and able to comply with all aspects of the treatment and evaluation schedule, and $\geq 1 \mathrm{~cm}$ of tumor-free lung parenchyma between target tumor and pleura or fissure (Figure 1).

Pre-procedure exclusion criteria included: contraindicated for surgery; prolonged infection of the chest, with the definition of this being lung consolidation that requires hospitalization and greater than 10 days of antibiotics 30 days prior to surgery; tumor abutting mainstem bronchus, main pulmonary artery branches, esophagus and/or trachea; tumor with pleural contact (as our primary endpoint was dose response and having it up against the pleura would distort the zones and exclusion decreased the risk of bronchopleural fistulas); tumors located $<3 \mathrm{~cm}$ from the staple lines/metal objects; subjects with stage IV emphysema per GOLD criteria; uncontrollable coagulopathy; inability to tolerate discontinuation of anti-coagulants prior to and during the ablation; pregnancy documented by a positive pregnancy test according to hospital standard practices) or actively breast-feeding; participation in an investigational drug or device research study within 30 days of enrollment that interferes with this protocol; the safety or welfare of the subject may be compromised by participation as determined by the investigator; and subjects with implantable pacemakers/other electronic implants (Figure 1). Intraprocedural exclusion criteria were an incidental finding that the subject no longer meets the study eligibility criteria (Figure 1).

\section{Statistical analysis}

Statistical analysis is described as follows. Intent-to-treat was defined as subjects enrolled into the study. Modified intent-to-treat was defined as intent to treat subjects who underwent the ablation procedure. Subject demographics and pre-treatment characteristics were summarized for the intent-to-treat population. The primary endpoint (analyses performed for modified intent-to-treat population) was dose response immediately post-procedure (for each target tumor) and was defined as the percentage change from the predicted outcome to the actual outcome. The percentage change [calculated as $100 \times(\mathrm{A}-\mathrm{P}) / \mathrm{P}$ ] was calculated for each target tumor. $\mathrm{P}$ represents predicted and A represents actual. For each tumor the percentage change 


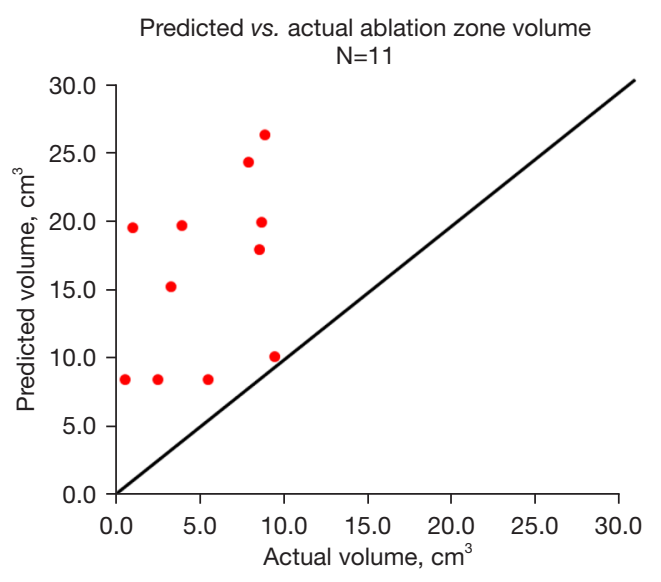

Figure 3 Predicted versus actual ablation zones. Predicted versus actual volume of ablation zone. Black line represents where Predicted Volume equals Actual Volume. For values above the lines, the ablation zone predicted by the software ablation zone was larger than the observed ablation zone. $\mathrm{N}=11$ and includes patients who had both predicted and CT volume measurements.

was calculated for ablation zone width $(X)$, ablation zone height $(\mathrm{Y})$, ablation zone depth $(\mathrm{Z})$, ablation zone volume, and ablation zone shape. For the measurements just described, the percentage change for all ablation zones was summarized. The predicted values were derived from the Emprint ${ }^{\mathrm{TM}}$ procedure planning application and the actual values came from the CT imaging. For secondary endpoints [analyses performed for modified intention to treat (MITT) population], the number and percentage of tumors that achieved complete tumor ablation, incomplete tumor ablation (delayed necrosis), and incomplete tumor ablation (positive margin) were presented. Complete ablation was assessed using histology. Histology was evaluated at a single center (National Jewish Health, Denver, CO, USA) by blinded pathologists. Safety Endpoints (analyses performed for MITT population) included incidence rates for adverse events and complications. Results of the study were expressed as mean \pm standard deviation, median with minimum and maximum listed, or percentage as depicted in the figures and tables of the manuscript.

\section{Results}

Nineteen adult subjects with metastatic or primary lung tumors consented to participate in the study. Ablation procedures were completed for 15 subjects, of which $15(100 \%)$ completed the study. Primary endpoints were available for 11 subjects of the original 15 (no post CT scans were obtained for one patient, post ablation CT images could not be read due to bleeding events for two patients, and one patient $C T$ analysis was missing a $Z$ (depth) reading. Of the original 15, 11 patients had secondary endpoints (no tumor was received in sample in 4 of the original 15 patients). Protocol deviations occurred in 5 subjects (26.3\%). Deviations included incomplete study assessment, study assessment/procedure not performed per protocol, and visit out of window timeframe. Additionally, two deviations were recorded in the 'other' category. In one case, the site used an imaging modality other than $\mathrm{CT}$ as specified in the protocol. In the second instance, an adjustment needed to be made in the device connection, causing an extension in general anesthesia time beyond the 20-30 minutes noted in the consent form.

The mean age for the 15 subjects that completed the study was 58.9 years. Sixty-seven percent were male; $33 \%$ were female. $33.3 \%(5 / 15)$ had a history of thoracic surgery, $73 \%(11 / 15)$ had metastatic tumors, and $27 \%(4 / 15)$ had primary tumors. Of those that had metastatic tumors, primary cancer types were colorectal $(33.3 \%)$, kidney (26.7\%), and other (12.2\%).

All 15 subjects underwent ablation and resection. The Emprint ${ }^{\mathrm{TM}}$ overestimated the volume of the ablation zone in all patients (Figure 3). In observing the percent difference between the observed outcome on post-ablation CT scan and the predicted outcome based on software prediction (primary endpoint), the percent difference in ablation zone width $(\mathrm{X})$ was $-43.6 \pm 18.8(\mathrm{n}=12)$, median: -44.7 (range, -77.4 to -11.2$)$; the percent difference in ablation zone height $(\mathrm{Y})$ was $-15.1 \pm 31.7(\mathrm{n}=12)$, median: -11.5 (range, -77.4 to 45.2 ); the percent difference in ablation zone depth (Z) was $-32.8 \pm 26.0(\mathrm{n}=11)$, median: -26.1 (range, -74.1 to 15.6); the percent difference in ablation zone volume (actual volume $v s$. predicted volume) was $-63.5 \pm 26.3(\mathrm{n}=11)$, median: -67.3 (range, -94.9 to -5.5 ); and the percent difference ablation zone volume (actual calculated volume vs. predicted volume) $-57.7 \pm 31.8(\mathrm{n}=11)$, median: -59.3 (range, -96.9 to 7.5 ) (Table 1). Based on histology (secondary endpoint), $54.5 \%$ (6/11) showed complete ablation, $36.4 \%$ (4/11) showed incomplete ablation, 9.1\% (1/11) showed delayed necrosis, and there was no tumor in sample for $36.1 \%$ (4/15) (Table 2; Figure 4). Table 3 outlines a summary of tumor location, diameter, and treatment statistics.

Twenty-one adverse events were reported for 11 subjects. There were no device-related adverse events. A total of 5 serious adverse events occurred in 3 subjects (20\%). 
Table 1 Primary endpoint: percentage difference between the observed outcome and predicted outcome based on immediate post-ablation CT scan

\begin{tabular}{lc}
\hline Variable & Mean \pm SD, median $(\min$, max $)$ \\
\hline \% Difference ablation zone width $(\mathrm{X}),(\mathrm{n}=12)$ & $-43.6 \pm 18.8,-44.7(-77.4,-11.2)$ \\
$\%$ Difference ablation zone height $(\mathrm{Y})(\mathrm{n}=12)$ & $-15.1 \pm 31.7,-11.5(-77.4,45.2)$ \\
$\%$ Difference ablation zone depth $(\mathrm{Z})(\mathrm{n}=11)$ & $-32.8 \pm 26.0,-26.1(-74.1,15.6)$ \\
$\%$ Difference ablation zone volume & $-63.5 \pm 26.3,-67.3(-94.9,-5.5)$ \\
$\begin{array}{l}\text { (actual volume vs. predicted volume) }(\mathrm{n}=11) \\
\text { (actual calculated volume vs. predicted volume) }(\mathrm{n}=12)\end{array}$ & $-57.7 \pm 31.8,-59.3(-96.9,7.5)$ \\
\hline
\end{tabular}

Table 2 Secondary endpoint: histology assessment for tumor ablation by subject

\begin{tabular}{lc}
\hline Variable & Percent of subjects (N=15 MITT) \\
\hline Complete ablation & $54.5 \%(6 / 11)$ \\
Incomplete ablation & $36.4 \%(4 / 11)$ \\
Delayed necrosis & $9.1 \%(1 / 11)$ \\
No tumor in sample & $36.4 \%(4 / 15)$ \\
\hline
\end{tabular}

MITT, modified intention to treat.

Serious adverse events included acute respiratory failure, pulmonary air leakage, prolonged air leak, Guillain Barre Syndrome, and respiratory arrest, which resolved the day after the procedure. All 5 serious adverse events were either not related $(n=2)$ or unlikely to be related $(n=3)$ to the ablation portion of the procedure (before the surgical wedge resection). There were no Grade $\geq 3$ adverse events. Twelve adverse events were related to the ablation procedure. The adverse events (as defined per CTCAE grading) included bleeding (Grade 1, n=1; Grade 2, n=3), pneumothorax (Grade 1, $\mathrm{n}=4$ ), hemoptysis (Grade 1, $\mathrm{n}=1$ ), hemothorax (Grade 2, n=2), and vasovagal syncope (Grade 1, n=1). All adverse events and serious adverse events resolved. There were no extended hospital stays.

\section{Discussion}

To our knowledge, this study was the first of its kind-an ablate and resect study in the lung designed to investigate software mapping for percutaneous microwave ablation followed by immediate tumor resection. Imaging immediately post procedure was used to measure dose response, while resected samples were used to assess histologically complete ablation. There were no devicerelated adverse events. The number and type of procedure related adverse events were expected and similar to those reported in literature.

On first pass, it appears that the Emprint ${ }^{\mathrm{TM}}$ procedure planning application grossly overestimates the amount of lung tissue that will be ablated as ablation zone volume measured on post ablation CT was $57.7 \%$ smaller than ablation zone size predicted by the software. However, using direct measurement of the observed ablation zone on postprocedure CT fails to account for the significant amount of tissue shrinkage that occurs during microwave ablation of lung tissue, which can be as high as $75 \%$ by volume (21). Tissue shrinkage alone can adequately explain the difference between predicted and observed ablation zone size, leaving open the question of how well the software predicts the amount of native lung that will be included in the ablation zone. It is critical that future studies using ablation zone size account for tissue shrinkage.

Regarding histologically complete ablation, our results are similar to previously published reports of (1-3). Histologically complete ablation was observed in $54.5 \%$ $(6 / 11)$ of subjects, with one subject having delayed necrosis. Of the four instances where incomplete ablation was noted, two may be merely due to the time the assessment was conducted rather than under-ablation, since continued destruction of tissue could continue as time goes by (21). The immediate time of examination to determine the area of ablation may have underestimated actual ablation than if more time had been given for this measurement (perhaps if the wedge resection had been performed a month or six months after the ablation). Outliers due to experimental error or limitations in methodology include in one instance that the tumor was not directly targeted, leading to an 

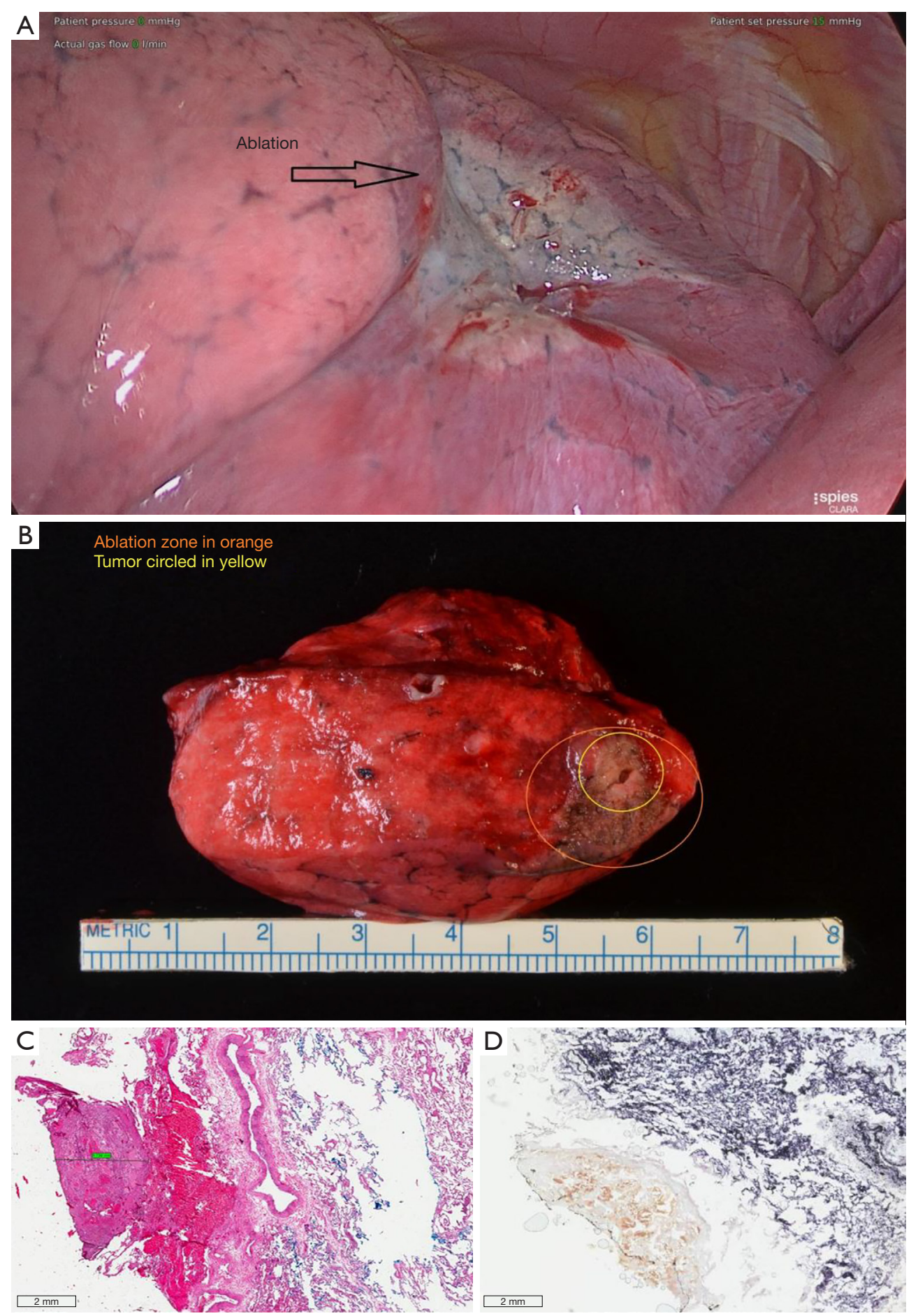

Figure 4 Ablation of tissue. (A) A representative image of intraoperative view of thoracoscopic evaluation of the ablation zone prior to wedge resection for the EMPrint ${ }^{\mathrm{TM}}$ Ablate and RESect Study in Patients with Metastatic Lung Tumors (EMPRESS) trial. (B) A representative image of the gross ablation zone and tumor. (C) Histology assessment: complete ablation; Hematoxylin and Eosin (H\&E) stain; high power. (D) Histology assessment: complete ablation; Hematoxylin and Eosin (H\&E); Nicotinamide adenine dinucleotide hydrogen (NADH) stain; high power. 
Table 3 Tumor location, diameter, and treatment statistics

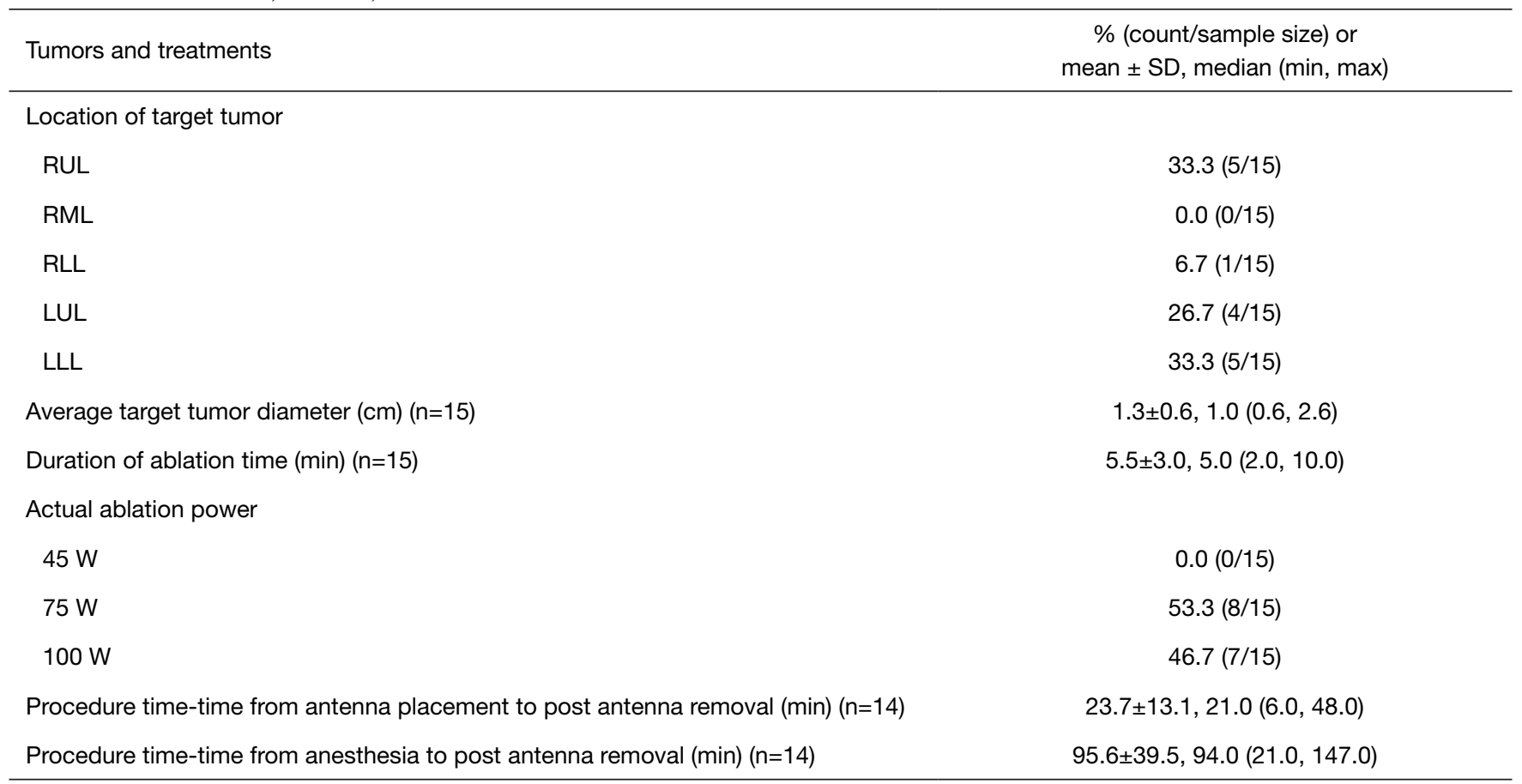

Tumors received one ablation. RUL, right upper lobe; RML, right middle lobe; RLL, right lower lobe; LUL, left upper lobe; LLL, left lower lobe.

ablation zone adjacent to the tumor. Sufficiency of targeting was measured by evaluating antenna placement relative to the tumor by CT imaging prior to the ablation. In one instance, the tumor was not directly targeted sufficiently, leading to an ablation zone adjacent to the tumor. In the second instance, after a thorough investigation by the study team, it was determined that an incorrect sample was obtained from a subject from whom multiple tumors were resected.

In this study, a percutaneous approach was utilized, compared to prior studies, which used an open thoracotomy approach. As there are limitations with previous ablate and resect studies that employed the open approach, there were several limitations in this study: First, it was difficult to obtain the ablated tumor in the resected sample (4/15). In 4/15 samples sent for study pathology, tumor was not observed. The study design limited the investigator to performing a single ablation, not allowing for re-activation after imaging assessment. Second, the aggressiveness of the ablation may have been influenced by the planned surgical procedure; smaller ablations may have been favored in order to not complicate the surgery to follow. Finally, imaging was the only measure for dose response and this imaging occurred immediately following the procedure. A high degree of inter-reader and intra-dose read variability may suggest difficulty with clear visualization of the margins of ablation zones on images taken immediately after the ablation procedure.

In summary, all enrolled subjects underwent CT-guided microwave ablation successfully, without any device-related adverse events, confirming the viability of an ablate and resect approach in the study of percutaneous ablation. Histologically complete ablation can be achieved with microwave ablation in the lung, but imaging with CT less than an hour after microwave ablation does not appear to be the ideal time point to measure dose response. In accordance with previous reports smaller ablation zones were expected, but tissue shrinkage was not accounted for and is a critical consideration in future studies when evaluating the accuracy of pre-procedure planning software and protocols (22-24). While a small dataset was gathered here, more robust studies in the lung are needed to describe dose-response in a clinical setting.

\section{Acknowledgments}

The paper was presented at the General Thoracic Surgical 
Club in Naples, Florida from March 7, 2019 to March 10, 2019.

Funding: The clinical trial was conducted and funded by Medtronic.

\section{Footnote}

Reporting Checklist: The authors have completed the TREND reporting checklist. Available at https://dx.doi. org/10.21037/jtd-21-594

Data Sharing Statement: Available at https://dx.doi. org/10.21037/jtd-21-594

Conflicts of Interest: All authors have completed the ICMJE uniform disclosure form (available at https://dx.doi. org/10.21037/jtd-21-594). RMS is an inventor on patents and royalties in the field of CAR-T cell therapy licensed to Humanigen through Mayo Clinic. JLP has leadership/ fiduciary roles and Stock/stock options in TMRW, Angiocrine Bioscience, and View Point Medical. DED has grants/contracts with Varian Inc.; royalties/licenses with Springer Verlag and Uptodate; consulting fees from Varian Inc; payment/honoraria from Moffitt Cancer Center; holds multiple patents (patent number 11076916 publication number 20190216537, patent number 10722289 publication number 20180153617, publication number 20200179049, publication number 20170182165); is the president and board member of Theromics Inc and the SIO program committee; and has stock/stock options with Theromics Inc. MRC has grants/contracts with Galil Medical, EDDA; royalties/licenses with Uptodate; consulting fees from Boston Scientific, Medtronic, Varian, and Pulse Biosciences; and a leadership/fiduciary role in the Society of Interventional Oncology. The other authors have no conflicts of interest to declare.

Ethical Statement: The authors are accountable for all aspects of the work in ensuring that questions related to the accuracy or integrity of any part of the work are appropriately investigated and resolved. The study was conducted in accordance with the Declaration of Helsinki (as revised in 2013). The study was approved by institutional review board of the participating institutions (IRB approval: 15-001772) on July 9, 2015 and informed consent was taken from all the patients.

Open Access Statement: This is an Open Access article distributed in accordance with the Creative Commons Attribution-NonCommercial-NoDerivs 4.0 International License (CC BY-NC-ND 4.0), which permits the noncommercial replication and distribution of the article with the strict proviso that no changes or edits are made and the original work is properly cited (including links to both the formal publication through the relevant DOI and the license). See: https://creativecommons.org/licenses/by-nc-nd/4.0/.

\section{References}

1. Dupuy DE. Image-guided thermal ablation of lung malignancies. Radiology 2011;260:633-55.

2. Mouli SK, Kurilova I, Sofocleous CT, et al. The Role of Percutaneous Image-Guided Thermal Ablation for the Treatment of Pulmonary Malignancies. AJR Am J Roentgenol 2017;209:740-51.

3. Wolf FJ, Aswad B, Ng T, et al. Intraoperative microwave ablation of pulmonary malignancies with tumor permittivity feedback control: ablation and resection study in 10 consecutive patients. Radiology 2012;262:353-60.

4. Vogl TJ, Naguib NN, Gruber-Rouh T, et al. Microwave ablation therapy: clinical utility in treatment of pulmonary metastases. Radiology 2011;261:643-51.

5. Yang X, Ye X, Huang G, et al. Repeated percutaneous microwave ablation for local recurrence of inoperable Stage I nonsmall cell lung cancer. J Cancer Res Ther 2017;13:683-8.

6. Zheng A, Wang X, Yang X, et al. Major complications after lung microwave ablation: a single-center experience on 204 sessions. Ann Thorac Surg 2014;98:243-8.

7. Wolf FJ, Grand DJ, Machan JT, et al. Microwave ablation of lung malignancies: effectiveness, CT findings, and safety in 50 patients. Radiology 2008;247:871-9.

8. Lu Q, Cao W, Huang L, et al. CT-guided percutaneous microwave ablation of pulmonary malignancies: Results in 69 cases. World J Surg Oncol 2012;10:80.

9. Vogl TJ, Worst TS, Naguib NN, et al. Factors influencing local tumor control in patients with neoplastic pulmonary nodules treated with microwave ablation: a risk-factor analysis. AJR Am J Roentgenol 2013;200:665-72.

10. Zheng A, Ye X, Yang X, et al. Local Efficacy and Survival after Microwave Ablation of Lung Tumors: A Retrospective Study in 183 Patients. J Vasc Interv Radiol 2016;27:1806-14.

11. Wei Z, Ye X, Yang X, et al. Microwave ablation in combination with chemotherapy for the treatment of advanced non-small cell lung cancer. Cardiovasc Intervent 
Radiol 2015;38:135-42.

12. Egashira Y, Singh S, Bandula S, et al. Percutaneous High-Energy Microwave Ablation for the Treatment of Pulmonary Tumors: A Retrospective Single-Center Experience. J Vasc Interv Radiol 2016;27:474-9.

13. Zhong L, Sun S, Shi J, et al. Clinical analysis on 113 patients with lung cancer treated by percutaneous CTguided microwave ablation. J Thorac Dis 2017;9:590-7.

14. Ko WC, Lee YF, Chen YC, et al. CT-guided percutaneous microwave ablation of pulmonary malignant tumors. J Thorac Dis 2016;8:S659-65.

15. Ratanaprasatporn L, Charpentier KP, Resnick M, et al. Intra-operative microwave ablation of liver malignancies with tumour permittivity feedback control: a prospective ablate and resect study. HPB (Oxford) 2013;15:997-1001.

16. Brace CL, Hinshaw JL, Laeseke PF, et al. Pulmonary thermal ablation: comparison of radiofrequency and microwave devices by using gross pathologic and CT findings in a swine model. Radiology 2009;251:705-11.

17. Dupuy DE. Microwave ablation compared with radiofrequency ablation in lung tissue-is microwave not just for popcorn anymore? Radiology 2009;251:617-8.

18. Vogl TJ, Eckert R, Naguib NN, et al. Thermal Ablation of Colorectal Lung Metastases: Retrospective Comparison Among Laser-Induced Thermotherapy, Radiofrequency Ablation, and Microwave Ablation. AJR Am J Roentgenol
2016;207:1340-9.

19. Menezes SL, Bozza PT, Neto HC, et al. Pulmonary and extrapulmonary acute lung injury: inflammatory and ultrastructural analyses. J Appl Physiol (1985) 2005;98:1777-83.

20. Pillai K, Akhter J, Chua TC, et al. Heat sink effect on tumor ablation characteristics as observed in monopolar radiofrequency, bipolar radiofrequency, and microwave, using ex vivo calf liver model. Medicine (Baltimore) 2015;94:e580.

21. Brace CL, Diaz TA, Hinshaw JL, et al. Tissue contraction caused by radiofrequency and microwave ablation: a laboratory study in liver and lung. J Vasc Interv Radiol 2010;21:1280-6.

22. Stern JM, Anderson JK, Lotan Y, et al. Nicotinamide adenine dinucleotide staining immediately following radio frequency ablation of renal tumors-is a positive stain synonymous with ablative failure? J Urol 2006;176:196972; discussion 1972.

23. Nguyen CL, Scott WJ, Young NA, et al. Radiofrequency ablation of primary lung cancer: results from an ablate and resect pilot study. Chest 2005;128:3507-11.

24. Akeboshi M, Yamakado K, Nakatsuka A, et al. Percutaneous radiofrequency ablation of lung neoplasms: initial therapeutic response. J Vasc Interv Radiol 2004; 15:463-70.
Cite this article as: Blackmon $\mathrm{SH}$, Sterner RM, Eiken PW, Vogl TJ, Pua BB, Port JL, Dupuy DE, Callstrom MR. Technical and safety performance of CT-guided percutaneous microwave ablation for lung tumors: an ablate and resect study. J Thorac Dis 2021;13(12):6827-6837. doi: 10.21037/jtd-21-594 


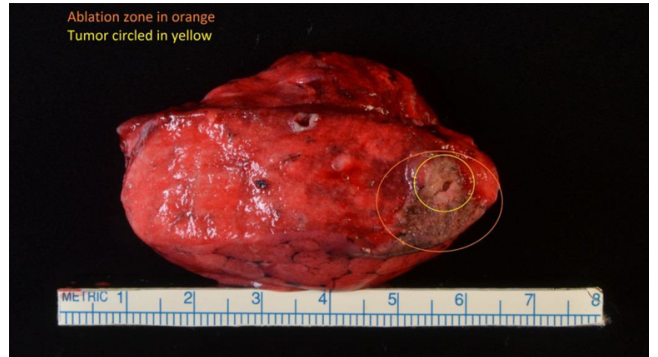

Figure S1 Lung wedge resection after pathologic processing to demonstrate the microwave ablated area with surrounding lung tissue after all stapled margins have been removed. The small circle represents the extent of the tumor and the larger circle represents ablated tissue markings from microwave energy.

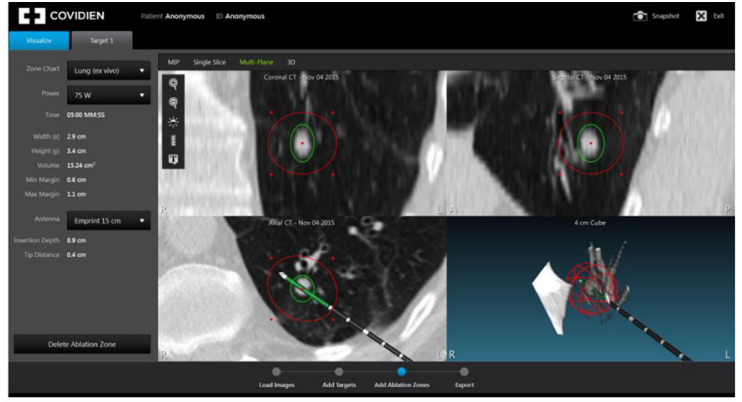

\section{Software planned probe placement.}
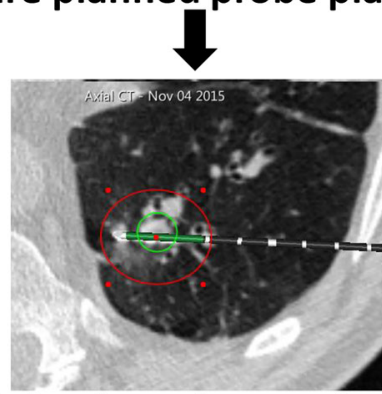

\section{Revised plan based on probe placement.}
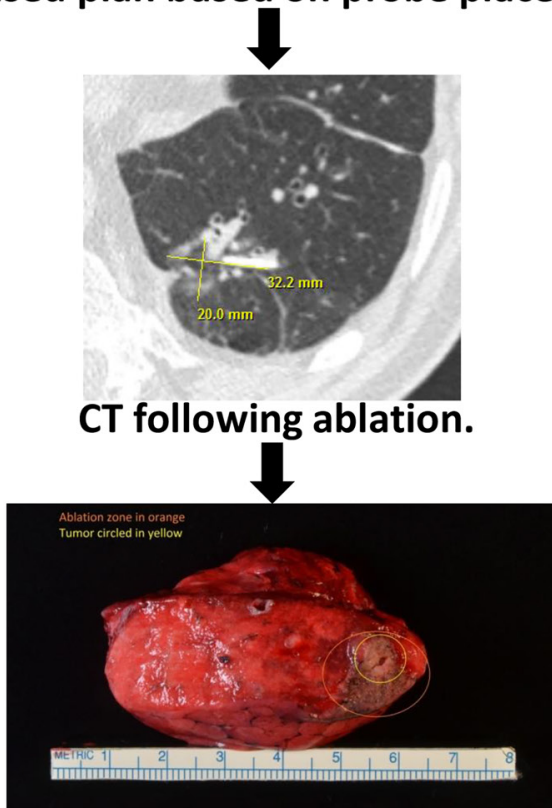

\section{Surgical resection and histological analysis.}

Figure S2 The stepwise approach for microwave tumor ablation including software planning for probe placement, revising probe position based on CT images, imaging the lesion after ablation, and finally, histologic analysis of the resected specimen. 